\title{
Frontières
}

\section{Le rapport à la maladie et le suicide chez les patients atteints de schizophrénie}

\section{Georgia Vrakas}

Volume 21, numéro 1, automne 2008

Prévenir le suicide

URI : https://id.erudit.org/iderudit/037875ar

DOI : https://doi.org/10.7202/037875ar

Aller au sommaire du numéro

\section{Éditeur(s)}

Université du Québec à Montréal

ISSN

1180-3479 (imprimé)

1916-0976 (numérique)

Découvrir la revue

Citer cet article

Vrakas, G. (2008). Le rapport à la maladie et le suicide chez les patients atteints de schizophrénie. Frontières, 21(1), 64-71. https://doi.org/10.7202/037875ar
Résumé de l'article

L'objectif de cette étude était d'examiner l'influence du rapport à la maladie sur le suicide chez les personnes atteintes de schizophrénie tel qu'il est reflété par la conscience, l'acceptation de la maladie et du traitement, l'acceptation et l'implication de la famille et les événements de vie associés à la maladie. La méthode de l'autopsie psychologique a été utilisée afin de comparer un groupe de 29 patients décédés par suicide et un groupe de 33 patients non suicidaires ayant un diagnostic de schizophrénie ou de trouble schizo-affectif. Les résultats indiquent que le risque de suicide augmente chez ceux ayant un rapport négatif à leur maladie, reflété par leur refus d'accepter la maladie, les événements de vie " générés " par la maladie et la dépression. Les stratégies de prévention devraient mettre l'accent sur l'amélioration de l'acceptation de la maladie des patients et sur le dépistage et le traitement adéquats de la dépression. 


\section{Résumé}

L'objectif de cette étude était d'examiner I'influence du rapport à la maladie sur le suicide chez les personnes atteintes de schizophrénie tel qu'il est reflété par la conscience, l'acceptation de la maladie et du traitement, l'acceptation et l'implication de la famille et les événements de vie associés à la maladie. La méthode de l'autopsie psychologique a été utilisée afin de comparer un groupe de 29 patients décédés par suicide et un groupe de 33 patients non suicidaires ayant un diagnostic de schizophrénie ou de trouble schizo-affectif. Les résultats indiquent que le risque de suicide augmente chez ceux ayant un rapport négatif à leur maladie, reflété par leur refus d'accepter la maladie, les événements de vie "générés" par la maladie et la dépression. Les stratégies de prévention devraient mettre l'accent sur l'amélioration de l'acceptation de la maladie des patients et sur le dépistage et le traitement adéquats de la dépression.

Mots clés: suicide - schizophrénie trouble schizo-affectif - conscience de la maladie - acceptation de la maladie - événements de vie.

\section{Abstract}

The goal of the study was to examine schizophrenic patients' relationship with their illness and its influence on completed suicide. To do so, we studied the impact of awareness of illness, of illness and treatment acceptance, of the family's acceptance and support and of illnessrelated events (i.e. those "generated" by the illness) on completed suicide. The study design was a case-controlled psychological autopsy study in which a group of 29 schizophrenic and schizoaffective patients who committed suicide were compared to a group of 33 living controls. The results indicate that a negative relation with one's illness does increase suicide risk as reflected by the refusal to accept one's illness, illnessrelated events and depression. Prevention strategies should focus on increasing illness acceptance and on the appropriate detection and treatment of depression.

Keywords: suicide - schizophrenia - schizoaffective disorder - illness awareness - illness acceptance - life events.

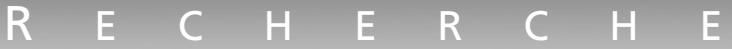

\section{Le rapport à la maladie et le suicide chez les patients atteints de schizophrénie}

\section{Georgia Vrakas, Ph. D., \\ chercheure postdoctorale, CRISE, Université du Québec à Montréal, CSSS de Bordeaux-Cartierville-Saint-Laurent, Centre affilié universitaire.}

La schizophrénie est un trouble de santé mentale grave et persistant affectant environ $1 \%$ de la population (Perälä et al., 2007). Les patients atteints de schizophrénie sont très à risque de se suicider. En effet, jusqu'à 42\% d'entre eux tenteront de se suicider au moins une fois au cours de leur vie (Caldwell et Gottesman, 1992) et entre 4\% (Inskip et al., 1998; Palmer et al., 2005) et 15\% (Amador et al., 1996) décéderont par suicide. L'objectif de cette étude était d'examiner l'impact du rapport à la maladie sur le suicide chez les patients ayant un diagnostic de schizophrénie.

\section{FACTEURS DE RISQUE DU SUICIDE}

La littérature fait référence à plusieurs facteurs de risque du suicide reflétant le rapport à la maladie chez les patients atteints de schizophrénie. Certains sont d'ordre clinique, telles les tentatives de suicide passées (Caldwell et Gottesman, 1992; De Hert et al., 2001; Haas, 1997; Kelly et al., 2005 ; Kuo et al., 2005 ; Rossau et Mortensen, 1997), les premières années suivant le diagnostic initial de la maladie (Kuo et al., 2005 ; Harkavy-Friedman et al., 1999), l'inadéquation du dosage de la médication prescrite ou la non-adhérence à celle-ci (De Hert et al., 2001; HarkavyFriedman et al., 1999), la présence de symptômes dépressifs (Hëila et al., 1997 ; Kelly et al., 2005; Kuo et al., 2005 ; Potkin et al., 2003; Rossau et Mortensen, 1997 ; Tejedor et al., 1987) souvent mal interprétée comme étant un symptôme négatif de la maladie par les professionnels de la santé (Saarinen et al., 1999), la conscience et l'acceptation de la maladie pour lesquels le lien avec le suicide n'est pas clairement établi. Certains chercheurs suggèrent que la conscience augmente le risque suicidaire (Amador et al., 1996; Pompili et al., 2004; Yen et al., 2002) et d'autres démontrent soit une diminution (Fenton, 2000), soit aucun impact sur le risque suicidaire (Bourgeois et al., 2004; Hawton et al., 2005; Recasens et al., 2002; Yen et al., 2002). Par ailleurs, aucune étude n'a été menée chez les patients atteints de schizophrénie ni sur son impact sur le suicide. Toutefois, la littérature portant sur d'autres maladies mentales graves et persistantes, tel le trouble bipolaire, a démontré que l'acceptation de la maladie est essentielle dans le maintien de la stabilité de la santé mentale chez ces patients (Greenhouse et al., 2000; Pollack, 1995 ; Russel et Browne, 2005). 
La littérature dégage aussi quelques facteurs de risque d'ordre écologique reflétant le rapport à la maladie chez les patients atteints de schizophrénie. Ces facteurs de risque de suicide sont les événements de vie (Harkavy-Friedman et al., 1999), plus précisément le type et la sévérité de ceux-ci (De Hert et al., 2001; Fennig et al., 2005; Hawton et al., 2005; Heilla et al., 1999) et certaines problématiques liées à la famille, telles que les conflits familiaux, l'attitude négative et la dysfonction familiale (Fennig et al., 2005; Giròn et Gòmez-Beneyto, 1998; King et Dixon, 1999; Miklowitz, 2004; Pompili et al., 2007).

\section{OBJECTIFS DE L'ÉTUDE}

Les écrits scientifiques nous informent donc que le risque de suicide est élevé chez les personnes atteintes de schizophrénie et que certains facteurs augmentent les risques de suicide. La littérature fait référence à plusieurs facteurs «internes», propres à la maladie et au patient, et à certains facteurs "externes » à l'individu. La majorité de ces facteurs reflètent la relation du patient face à sa maladie, spécifiquement le cours de la maladie, la prise de médication, la conscience de la maladie et l'acceptation de celle-ci; certains autres considèrent le rapport des autres face à la maladie (la famille et les événements de vie).

Cependant, il n'y a pas de consensus concernant la nature du rôle de la conscience de la maladie sur le suicide complété. Plusieurs questions restent sans réponse: est-ce que la conscience de la maladie - la capacité du patient à être conscient du fait qu'il est malade/qu'il a un diagnostic de trouble de santé mentale est nécessaire pour que la personne puisse améliorer son état de santé mentale? La conscience de la maladie protège-t-elle la personne contre le suicide ou au contraire augmente-t-elle le risque de suicide? Par ailleurs, aucune étude n'a investigué le rôle de l'acceptation de la maladie et le suicide chez les patients atteints de schizophrénie. En revanche, des études ont clairement établi l'importance de l'acceptation de la maladie et de la réadaptation positive, ainsi que de la stabilité mentale chez les personnes atteintes de troubles bipolaires. Il y a très peu d'informations sur l'influence des événements de vie sur le suicide complété, sur le rôle de la famille et le suicide chez ces patients; la littérature suggère toutefois que ces facteurs peuvent augmenter le risque de suicide chez ces patients.

Le but de cette étude était donc d'examiner l'influence du rapport à la maladie sur le suicide chez les personnes atteintes de schizophrénie telle qu'elle est reflétée par: la conscience, l'acceptation de la maladie et la période durant laquelle a lieu le suicide; l'acceptation et l'implication de la famille, ainsi que les événements de vie, plus particulièrement ceux associés à la maladie (hospitalisations, problèmes avec la justice, perte d'emploi, etc.).

\section{HYPOTHÈSES}

Plus précisément, nous avons prédit que :

1. les patients atteints de schizophrénie décédés par suicide (cas) seraient moins conscients de leur maladie que les patients schizophrènes non décédés et non suicidaires (groupe témoin);

2. les patients décédés par suicide refuseraient d'accepter leur maladie plus souvent que les patients non suicidaires;

2.1 les patients décédés par suicide refuseraient leur traitement médicamenteux plus souvent que les patients du groupe non suicidaire;

3. le risque de suicide serait plus élevé durant les 10 premières années suivant le diagnostic;

4. une plus grande proportion des parents dans le groupe suicide n'accepteraient pas la maladie de leur enfant au moment du diagnostic comparativement aux parents des patients non suicidaires;

5. les parents des patients décédés par suicide seraient moins impliqués dans le traitement de la maladie de leur enfant au moment du décès comparativement aux parents des patients du groupe non suicidaire;

6. les événements de vie seraient plus souvent associés à la maladie chez les patients décédés par suicide que chez les patients non suicidaires.

\section{MÉTHODOLOGIE}

Pour réaliser les objectifs de cette étude, la méthode de l'autopsie psychologique a été utilisée, une méthode rétrospective d'étude permettant la collecte de données sur la santé psychologique et physique de la personne décédée par suicide, sur son enfance et des événements de vie en interviewant des membres de la famille (Pouliot et De Leo, 2006).

\section{PARTICIPANTS ET RÉPONDANTS}

Deux groupes de participants pairés selon le sexe, l'âge et le diagnostic ont été comparés. Le groupe suicide (cas) était composé de 23 hommes et de 6 femmes décédés par suicide entre 1997 et 2004 . Vingt-sept d'entre eux avaient un diagnostic de schizophrénie et deux avaient un diagnostic de trouble schizo-affectif. Les entrevues ont eu lieu entre 4 mois et 5 ans suivant le décès (moyenne: 2 ans et 7 mois). Les cas ont été recrutés à travers le Bureau du coroner du Québec, la Société québécoise de schizophrénie (SQS) et la Fédération des familles et amis de la personne atteinte de maladie mentale (FFAPAMM).

Le groupe témoin était composé de 33 patients non suicidaires (selon l'évaluation de leurs psychiatres) dont 24 hommes et 9 femmes. Vingt-huit avaient un diagnostic de schizophrénie et cinq avaient un diagnostic de trouble schizoaffectif. Ces patients ont été recrutés à travers la Clinique des jeunes adultes de l'Hôpital Louis-H.-Lafontaine, la Clinique communautaire de Verdun, l'Institut Allan Memorial (Centre universitaire de santé de l'Université McGill), la SQS et la FFAPAMM.

Des patients avec un diagnostic de trouble schizo-affectif ont été inclus à cause de la difficulté de recruter des patients schizophrènes seulement. Cette inclusion ne pose pas problème puisque les facteurs de risque de suicide sont similaires chez ces deux groupes (Potkin et al., 2003).

Dans les deux groupes, les entrevues ont été menées auprès d'un membre de la famille connaissant bien le participant. La majorité des répondants étaient des mères (plus de 50\%), suivi d'un membre de la fratrie.

\section{INSTRUMENTS DE MESURE}

\section{INFORMATIONS}

SOCIODÉMOGRAPHIQUES

La première section du K-SADS-E (Puig-Antich et al., 1995), adapté et traduit en français, a été utilisée pour la collecte des données sociodémographiques.

\section{DIAGNOSTICS PSYCHIATRIQUES}

Les diagnostics psychiatriques des cas et des patients ont été déterminés à l'aide de versions adaptées (à l'utilisation avec une tierce personne) du SCID I pour les troubles de l'Axe I (First et al., 2001) et le SCID II pour les troubles de l'Axe II (First et al., 1995) ainsi qu'à l'aide des dossiers médicaux lorsque cela était possible. Les diagnostics finaux étaient déterminés par un panel de cliniciens (accord interjuges variant entre 0,72 et 1,0$)$.

\section{CONSCIENCE, ACCEPTATION DE LA MALADIE ET ACCEPTATION FAMILIALE}

Plusieurs instruments existants mesurent le niveau de conscience de la maladie (Amador et al., 1994; Bourgeois et al., 2004; Yen et al., 2002; Recasens et al., 2002) mais aucun n'a été utilisé ni validé dans le cadre d'autopsies psychologiques. Par ailleurs, nous voulions évaluer l'acceptation de la maladie par le patient et par sa famille. Pour ces raisons, 
un instrument évaluant la conscience de la maladie, l'acceptation de la maladie et du traitement ainsi que l'acceptation de la maladie par la famille et l'implication de la famille dans le traitement de leur proche a été développé.

Afin de déterminer si les questions que nous avons créées concernant la conscience de la maladie, l'acceptation de la maladie et du traitement médicamenteux et l'acceptation et l'implication de la famille dans le traitement de leur proche mesuraient réellement la conscience de la maladie du patient, son acceptation de sa maladie et de son traitement et l'acceptation de la maladie et l'implication dans le traitement par sa famille, nous avons établi la validité concomitante de ces items. Ceci a été accompli en passant le même questionnaire à un autre proche qui connaissait bien la personne décédée dans cinq cas de suicide. Dans le groupe témoin, nous avons interviewé le patient lui-même dans six cas. Des corrélations de Pearson ont été effectuées avec les réponses de chaque paire de répondants pour chacun des deux groupes de patients (suicide et témoin). Les résultats ont révélé des corrélations significatives pour chacun des items (de 0,67 à 1,0). L'accord interjuges a aussi été établi et était élevé pour tous les items du questionnaire (de 0,87 à 1,0).

\section{ÉVÉNEMENTS DE VIE}

Le «Life Events and Difficulties Schedule» (LEDS; Brown et al., 1989) a été utilisé afin de mesurer les événements vécus durant les douze derniers mois. Dans la présente étude, nous étions particulièrement intéressées par les événements de vie associés à la maladie (hospitalisations, déménagement en foyer de groupe, divorce/séparation due à la maladie, retour forcé de voyage à cause de la maladie, etc.).

\section{RÉSULTATS}

Les données ont été analysées avec des tests t de Student, chi-carré de Pearson, test exact de Fisher et des régressions logistiques à l'aide du logiciel SPSS (version 11.0). Le seuil de significativité était considéré à $\mathrm{p} \leq 0,05$.

\section{DESCRIPTION DES PARTICIPANTS -}

\section{DONNÉES SOCIODÉMOGRAPHIQUES}

Les participants des deux groupes étaient similaires sur le plan des variables sociodémographiques étudiées: âge, niveau de scolarité, emploi, statut marital et résidence (voir tableau 1).

\section{CONSCIENCE, ACCEPTATION}

DE LA MALADIE ET DU TRAITEMENT

La première hypothèse qui prédisait que les cas du groupe suicide seraient moins

TABLEAU 1

DONNÉES SOCIODÉMOGRAPHIQUES

\begin{tabular}{lcc}
\hline & Groupe suicide & Groupe témoin \\
Âge moyen (années) & 31,7 & 34,9 \\
Niveau de scolarité (années) & 11,28 & 12,33 \\
Emploi $(n=)$ & & \\
$\quad$ Sans emploi & $74 \%(20)$ & $69 \%(20)$ \\
$\quad$ Bénévolat & $4 \%(1)$ & $3 \%(1)$ \\
$\quad$ Travail et/ou études & $22 \%(6)$ & $28 \%(8)$ \\
Statut marital $(n=)$ & & $3,4 \%(1)$ \\
$\quad$ Séparé/divorcé & $17,2 \%(5)$ & $18,2 \%(6)$ \\
$\quad$ Marié/conjoint de fait & $3,4 \%(1)$ & \\
Résidence $(n=)$ & & $25 \%(8)$ \\
$\quad$ Vivant seul & $29 \%(8)$ & $31 \%(10)$ \\
$\quad$ Vivant avec parents & $43 \%(12)$ & $16 \%(5)$ \\
$\quad$ Vivant en foyer de groupe & $25 \%(7)$ & \\
\hline
\end{tabular}

conscients de leur maladie que les patients du groupe témoin n'a pas été confirmée. En effet, il n'y avait aucune différence entre le groupe suicide et le groupe témoin sur le plan de la conscience de la maladie. Soixante-dix-neuf pour cent des patients du groupe suicide et $88 \%$ des patients du groupe témoin étaient conscients de leur maladie $(\mathrm{p}=0,263)$.

La deuxième hypothèse prédisant que les patients du groupe témoin accepteraient leur maladie significativement plus souvent que les patients du groupe suicide a été confirmée. Soixante et onze pour cent des patients non suicidaires acceptaient leur maladie comparativement à $15 \%$ seulement du groupe suicide $\left(\chi^{2}=17,15, d f=1\right.$, $p=0,000)$. Par ailleurs, $58 \%(11 / 19)$ des personnes décédées par suicide qui avaient refusé leur maladie avaient indiqué que ce refus était à l'origine de pensées et comportements suicidaires contre $15 \%(5 / 33)$ des patients dans le groupe témoin $\left(\chi^{2}=11,69\right.$, $d f=2, p=0,003)$.

En ce qui concerne le traitement de la maladie, les patients du groupe témoin acceptaient leur traitement médicamenteux dans une proportion significativement plus importante que le groupe suicide $(82 \%$ contre $\left.46 \% ; \chi^{2}=8,41, d f=1, p=0,004\right)$.

\section{NOMBRE D'ANNÉES VÉCUES \\ AVEC LE DIAGNOSTIC}

La troisième hypothèse, prédisant que le risque de suicide serait plus élevé durant les 10 premières années suivant le diagnostic, a été confirmée. Le nombre d'années vécues avec le diagnostic variait entre 1 à 30 ans avec une moyenne de 7,5 ans (médiane: 4 ans). Les diagnostics étaient reçus en moyenne à l'âge de 24,2 ans (entre 12 et 45 ans) et l'âge moyen au moment du décès était de 31,7 ans (entre 20 et 56 ans).

\section{ACCEPTATION ET IMPLICATION \\ DE LA FAMILLE}

La quatrième hypothèse portant sur une acceptation de la maladie par la famille plus importante chez les patients du groupe témoin n'a pas été confirmée. Comme nous n'avions que peu de données sur les pères et mères séparément (12 et 16 respectivement, au total), une nouvelle catégorie a été créée: "au moins un membre de la famille accepte la maladie ». Les résultats indiquent qu'au moins un membre de la famille acceptait la maladie chez 14 cas $(52 \%)$ dans le groupe suicide et chez 16 patients $(62 \%)$ dans le groupe témoin $\left(\chi^{2}=0,51 ; d f=1 ; p=0,477\right)$.

La cinquième hypothèse prédisant que plus de familles de patients du groupe témoin seraient impliquées dans le traitement de leur proche n'a pas été confirmée. Il n'y avait aucune différence dans les groupes : dans le groupe suicide, 89\% (24) des cas avaient au moins un membre de leur famille impliqué dans leur traitement et, dans le groupe témoin, cette proportion était similaire avec 84\% (26) $(p=0,435)$.

\section{ÉVÉNEMENTS DE VIE}

La dernière hypothèse prédisant plus d'événements de vie associés à la maladie chez les patients du groupe suicide que chez ceux du groupe témoin a été confirmée. En effet, le nombre moyen d'événements de vie associés à la maladie était significativement plus élevé chez les cas du groupe suicide $(2,64)$ que chez les patients du groupe témoin $(0,68)(t=4,81 ; p=0,008)$. De plus, la grande majorité de ces événements liés à la maladie étaient sévères $(91 \%)$ chez les patients du groupe suicide contre seulement $42 \%$ chez les patients du groupe témoin. En ce qui concerne la nature des événements associés à la maladie vécus au cours des 12 derniers mois, les résultats 
indiquent que les cas du groupe suicide avaient été hospitalisés (ou hébergés en centre de crise) significativement plus souvent que ceux du groupe témoin $(t=3,72$; $p=0,000$ ). De plus, les patients décédés avaient vécu significativement plus d'événements reflétant une détérioration marquée $(p=0,000)$, et plus d'événements de vie impliquant de la violence $(p=0,000)$ que les patients du groupe témoin. Par ailleurs, les événements de vie associés à la maladie étaient significativement corrélés au refus d'accepter la maladie $(r=0,345 ; p \leq 0,05)$. Nous avons aussi comparé le nombre total d'événements de vie (peu importe leur nature) entre les deux groupes. Les résultats indiquent que les deux groupes avaient vécu un nombre élevé d'événements de vie associés à la maladie au cours des douze derniers mois: 6,24 chez les patients décédés et $6,23 \mathrm{chez}$ les patients vivants $(t=0,01, p=0,989)$.

\section{COMORBIDITÉ}

Des analyses secondaires ont révélé que le groupe suicide avait un nombre significativement plus élevé de troubles de l'Axe I actuels que le groupe témoin (1,69 contre $1,23 ; p=0,023)$. Il n'y avait aucune différence de groupe sur le plan de l'abus et de la dépendance à l'alcool ou à d'autres substances. Il y avait, par contre, une différence significative concernant la dépression non spécifiée présente $(p=0,012): 26 \%(7)$ du groupe suicide qui présentait ce diagnostic contre 3\% (1) seulement du groupe témoin. De plus, la dépression non spécifiée présente était négativement corrélée à l'acceptation de la maladie $(r=-0,354$; $p=0.017)$.

\section{PRÉDICTEURS DU SUICIDE}

Une analyse de régression logistique hiérarchique a fait ressortir deux variables qui prédisaient significativement le suicide : le refus d'accepter la maladie $(O R=10,106$; $95 \%$ CI: 2,085 - 48,986) et les événements de vie associés au suicide $(O R=1,898$; $95 \% C I: 1,128$ - 3,195).

\section{DISCUSSION}

\section{FACTEURS INTERNES}

ET RISQUES DE SUICIDE

Les résultats indiquent qu'il n'y a aucun effet de la conscience de la maladie sur le suicide chez les patients atteints de schizophrénie. Cela appuie les résultats de certaines études (Hawton et al., 2005; Recasens, et al., 2002; Yen et al., 2002) qui n'ont trouvé aucun lien entre la conscience de la maladie et le risque suicidaire, l'anxiété ni la dépression. Par ailleurs, Bourgeois et collègues (2004) suggèrent que la dépression, et non la conscience

de la maladie, augmente le risque de suicide. Kim, Jayathilake et Meltzer (2003) ont trouvé que la conscience de la maladie peut augmenter le risque de suicide mais seulement si le désespoir est présent. Nous avons trouvé que la dépression était significativement associée au suicide mais nous n'avons pas examiné le rôle du désespoir sur le suicide chez les patients atteints de schizophrénie. Cette relation reste un sujet à investiguer davantage chez ces patients vulnérables.

Le refus d'accepter la maladie prédit le suicide. Par ailleurs, le refus d'accepter la maladie est corrélé à la dépression non spécifiée actuelle. Cela semble indiquer que ce facteur contribue, au moins en partie, au développement de la dépression chez les personnes atteintes de schizophrénie. Nous avons aussi trouvé que les premières années suivant le diagnostic initial constituent la période la plus à risque de suicide et, selon la littérature, le début du développement de la maladie et les rechutes sont les périodes les plus à risque pour le développement de la dépression (Häfner, 2005; Häfner et al., 2005a; Häfner et al., 2005b). Nos résultats indiquent que les patients

NOUS N'AVONS PAS TROUVÉ D'ASSOCIATION SIGNIFICATIVE ENTRE LA CONSCIENCE DE LA MALADIE EN TANT QUE TELLE ET LE SUICIDE. CEPENDANT, CE QUI RESSORT CLAIREMENT EST LA SOUFFRANCE RESSENTIE PAR LES PATIENTS SUICIDÉS, CAUSÉE PAR LES PERTES QU'ILS ONT VÉCUES — NON PAS LA PERTE D'AMIS, LE REJET PAR LEUR FAMILLE, MAIS PLUTÔT LA PERTE DE LEUR AVENIR, DE LEUR CARRIÈRE POTENTIELLE ET DE LEURS RELATIONS AMOUREUSES POTENTIELLES.

décédés par suicide étaient plus souvent hospitalisés au cours des douze derniers mois comparativement au groupe témoin, ce qui suggère la présence de rechutes et, par conséquent, une augmentation de la dépression.

En ce qui concerne l'acceptation du traitement médicamenteux, les résultats indiquent que les patients qui refusent leurs médicaments, donc, qui ne prennent pas leur médication telle que prescrite par leur médecin, sont plus à risque de se suicider. Cela s'appuie sur la littérature qui a démontré que les patients schizophrènes décédés par suicide ont plus tendance à ne pas adhérer à leur médication que l'ensemble des patients atteints de schizophrénie (De Hert et al., 2001; Hunt et al., 2006; Leucht et Heres, 2006; Ward et al., 2006).

\section{FACTEURS EXTERNES}

ET RISQUES DE SUICIDE

L'acceptation et l'implication de la famille n'ont aucun effet significatif sur le suicide chez les patients atteints de schizophrénie. La famille, pour la majorité, accepte la maladie et est impliquée dans le traitement du proche malade. Pourtant, cela n'agit pas comme facteur protecteur. En ce qui concerne les événements de vie, leur nombre n'a pas d'impact sur le risque suicidaire. Ces résultats appuient ceux obtenus de certaines études passées (Heïla et al., 1999). Ce sont plutôt les événements de vie associés à la maladie, pour la majorité sévères, qui augmentent le risque de suicide chez ce groupe psychiatrique. De plus, ces événements sont corrélés au refus d'accepter la maladie. Ces résultats suggèrent donc que ce sont plutôt des facteurs internes à l'individu qui augmenteraient le risque suicidaire, car même les facteurs dits externes - les événements de vie - qui ont un impact significatif sur le suicide, émergent de l'individu, dans le sens qu'ils sont "générés» par la maladie et le refus de l'accepter. 
causé par le psymal, une souffrance psychologique extrême que la personne ne peut plus tolérer, qu'elle tente de fuir en se suicidant. Selon Apter et Ofek (2001), une personne atteinte de maladie mentale, telle que la schizophrénie, vivra une démoralisation, du désespoir et de la dépression causés par la maladie. En effet, le désespoir est une caractéristique importante chez les personnes qui se suicident (Mishara et Tousignant, 2004) et un facteur qui augmente le risque du suicide chez les personnes atteintes de schizophrénie (Chan-Hyung et al., 2003 ; Kaneda, 2005) même lorsqu'on tient compte de la dépression (Bolton et al., 2007). Nous n'avons pas spécifiquement étudié le désespoir dans la présente étude; cependant, nos résultats reflètent la souffrance interne, la détresse que les patients suicidés avaient exprimée à leurs proches. On devrait davantage explorer l'impact du désespoir sur le suicide chez les patients atteints de schizophrénie.

Breed (1967) fait le lien entre la conscience d'avoir perdu son emploi, sa conjointe, sa position dans la société et le suicide. Nous n'avons pas trouvé d'association significative entre la conscience de la maladie en tant que telle et le suicide. Cependant, ce qui ressort clairement est la souffrance ressentie par les patients suicidés, causée par les pertes qu'ils ont vécues - non pas la perte d'amis, le rejet par leur famille, mais plutôt la perte de leur avenir, de leur carrière potentielle et de leurs relations amoureuses potentielles. Dans plusieurs cas, les personnes avaient mentionné ne plus avoir de but dans la vie, avoir une vie sans avenir, ne pas avoir d'emploi, ne pas être normal, avoir une faible estime de soi, se sentir humilié par leur diagnostic, être incapable d'accepter la maladie mentale comme faisant partie d'eux. Ils semblent donc très conscients de ces pertes, de ces changements dans leur vie mais ils sont incapables de s'ajuster à leur maladie, de l'accepter, ce qui se reflète clairement par le refus d'accepter leur maladie et son traitement et par la détresse exprimée précédant leur suicide. Selon la littérature, les sentiments négatifs face à la maladie et face à soi-même, le sentiment de désespoir, le pessimisme exprimé face à la maladie et l'humiliation ressentie ont un impact sur la dépression (Williams, 1997 ; Birchwood et al., 1993 ; Birchwood et Chadwick, 1997 ; Rooke et Birchwood, 1998) et les idéations suicidaires (Fialko et al., 2006) chez les personnes atteintes de schizophrénie. Ainsi, nos résultats montrent que le rapport négatif à la maladie, plutôt que la maladie en tant que telle, augmente le risque de suicide.

\section{LIMITES ET FORCES DE L'ÉTUDE}

Cette étude présente quelques limites. Premièrement, l'autopsie psychologique est de nature rétrospective: les patients sont décédés et donc inaccessibles. Les données ont été collectées par des entrevues menées auprès d'un tiers. Dans la majorité des cas, nous n'avions qu'un seul répondant, ce qui peut limiter la quantité et la qualité de l'information obtenue. Cependant, lorsque nous n'avions pas suffisamment d'information sur la personne décédée, nous avions accès à son dossier médical/psychiatrique. Par ailleurs, nous avons suivi les recommandations de plusieurs auteurs concernant l'autopsie psychologique: les groupes (suicide et témoin) étaient pairés selon l'âge, le sexe et le diagnostic (Hawton et al., 1998; Isometsa, 2001); les répondants étaient des membres de la famille connaissant très bien le participant, et ce, dans le groupe suicide et le groupe témoin (Hawton et al., 1998) ; les instruments diagnostiques utilisés (SCID I et SCID II) dans la collecte de données avaient déjà été exploités auparavant dans d'autres études d'autopsies psychologiques (Lesage et al., 1994; McGirr et al., 2006).

Une autre limite de cette étude est la petite taille de l'échantillon (29 cas dans le groupe suicide et 33 patients dans le groupe témoin). Cependant, la schizophrénie est un trouble mental rare et le suicide est un phénomène relativement rare: la mort par suicide a lieu chez 5\% des personnes atteintes de schizophrénie (Inskip et al., 1998; Palmer et al., 2005). Il est donc difficile de trouver dans la population des gens ayant ce diagnostic et qui sont décédés par suicide. Le recrutement des patients décédés par suicide a duré cinq ans. Le recrutement des patients atteints de schizophrénie, décédés par suicide dont les proches acceptent de participer à l'étude est difficile, aussi à cause de la nature stigmatisée de la maladie mentale grave et de la mort par suicide. En tenant compte de tout cela, la taille de l'échantillon est acceptable et représentative de la population atteinte de schizophrénie (ou autres troubles psychotiques) et décédée par suicide.

Cette étude a plusieurs forces, dont l'utilisation du Life Events and Difficulties Schedule (Brown et al., 1989). Le LEDS, une entrevue semi-structurée sur les événements de vie et des difficultés ayant eu lieu durant les douze derniers mois, a permis une collecte de données riches d'ordre qualitatif, ce qui n'est pas le cas d'autres instruments structurés de type "items à cocher» utilisés dans plusieurs autres études sur le suicide et la schizophrénie (Fennig et al., 2005; Heïla et al., 1999).
Une autre force de cette étude est le développement et la validation du questionnaire sur la conscience et l'acceptation de la maladie. Cet outil permet l'évaluation de la conscience et l'acceptation de la maladie du patient décédé via l'entrevue auprès d'un tiers, ce qui n'est pas le cas des outils existants évaluant seulement la conscience de la maladie du patient (Amador et al., 1994; Recasens et al., 2002; Yen et al., 2002; Bourgeois et al., 2004). Il permet aussi l'examen du lien entre le refus de l'acceptation de la maladie et les pensées et comportements suicidaires De plus, cet instrument évalue l'acceptation de la maladie par la famille et l'implication de la famille.

\section{RETOMBÉES POUR LA PRÉVENTION ET L'INTERVENTION}

Les résultats de cette étude suggèrent plusieurs pistes de prévention et d'intervention auprès de personnes atteintes de schizophrénie et de leurs familles.

\section{AUGMENTATION DE L'ACCEPTATION \\ DE LA MALADIE}

Premièrement, les résultats indiquent clairement que le refus d'accepter la maladie a un effet significatif sur le risque de suicide chez ces patients. Les professionnels de la santé œuvrant auprès de cette clientèle devraient travailler davantage l'acceptation de la maladie et du traitement avec leurs patients étant donné l'impact que cela peut avoir sur la diminution du risque suicidaire. De plus, nos résultats indiquent que le refus d'accepter la maladie est corrélé aux événements de vie sévères associés à la maladie et à la dépression non spécifiée. En développant des stratégies d'acceptation de la maladie, une diminution des événements de vie générés par la maladie et de la dépression pourrait s'ensuivre.

\section{LUTTE CONTRE LA STIGMATISATION}

L'acceptation de la maladie par le patient peut être, cependant, un processus difficile étant donné la stigmatisation associée à la maladie mentale, surtout à la schizophrénie (Phelan et al., 2000). La stigmatisation peut être définie comme un attribut de la personne (des comportements bizarres, avoir eu un contact avec un psychiatre, un diagnostic, etc.) qui fait de celle-ci quelqu'un de différent des autres, d'indésirable et d'anormal (Sartorius, 2007 ; Goffman, 1986, dans Schulze et Angermeyer, 2003). En effet, le public a une perception de la schizophrénie et de la psychose très négative et perçoit les gens qui en souffrent comme étant dangereux, violents et agressifs (Shulze et Angermeyer, 2003 ; Phelan et al., 2000). 
LES RÉSULTATS SUR LES ÉVÉNEMENTS DE VIE ASSOCIÉS À LA MALADIE

SOULÈVENT L'IMPORTANCE DU CONTACT AVEC DES PROFESSIONNELS

DE LA SANTÉ PRÉCÉDANT LE SUICIDE : LES PATIENTS DÉCÉDÉS PAR

SUICIDE AVAIENT EU UN NOMBRE SIGNIFICATIVEMENT PLUS ÉLEVÉ

D'HOSPITALISATIONS OU DE SÉJOURS EN CENTRE DE CRISE AU COURS

DES DOUZE DERNIERS MOIS QUE LES PATIENTS DU GROUPE TÉMOIN.

CELA SUGGÈRE QUE LES PATIENTS SUICIDÉS AVAIENT UN NOMBRE

IMPORTANT DE CONTACTS AVEC DES PROFESSIONNELS

DE LA SANTÉ DURANT LA DERNIÈRE ANNÉE DE LEUR VIE.

Cette perception négative peut aussi être présente chez les patients atteints de schizophrénie qui risquent d'avoir des connaissances confuses sur leur maladie, influencées par les informations diffusées par les médias et par les attitudes négatives des gens face à la schizophrénie (Schulze et Angermeyer, 2003). Cela peut faire en sorte de rendre l'acceptation de leur diagnostic psychiatrique difficile d'autant plus qu'ils se trouvent à perdre leurs amis, à perdre leur emploi, à souffrir d'effets secondaires des médicaments, à faire le deuil de leurs rôles sociaux potentiels (époux, parent, professionnel) et à avoir une identité sociale qui leur est «imposée», c'est-à-dire celle d'un malade fou, agressif et violent (Schulze et Angermeyer, 2003).

Des programmes existent pour réduire la stigmatisation face à la maladie mentale, dont le programme international pour lutter contre la stigmatisation et la discrimination des personnes atteintes de schizophrénie du World Psychiatric Association (WPA) implanté dans 19 pays dont le Canada, l'Alberta étant le site pilote pour la campagne internationale contre la stigmatisation des personnes atteintes de schizophrénie (voir le site: <http:// www.open-the-doors.com/ $>$ ). Le but de ce programme international de type éducatif est d'améliorer les connaissances des gens sur la schizophrénie et des différents traitements existants. L'accent est mis sur les aspects sociaux de la maladie, dont la stigmatisation et la discrimination face aux personnes atteintes de cette maladie (Fleischhacker et al., 1998). Les résultats du programme albertain semblent prometteurs concernant les interventions qui visent des sous-populations spécifiques (ex.: étudiants à l'école secondaire) qui privilégient les contacts directs avec les personnes atteintes de schizophrénie (Stuart, 2003). En effet, une amélioration des connaissances sur la schizophrénie et des attitudes face aux personnes atteintes de cette maladie a été démontrée chez les jeunes des écoles secondaires exposés à ce programme (Stuart, 2003).

La réduction de la stigmatisation et de la discrimination face aux personnes souffrant de schizophrénie via des programmes d'éducation comme celui du WPA présente une voie intéressante: en changeant les attitudes du public, dont les personnes atteintes de schizophrénie font partie, on pourrait aussi agir sur les perceptions qu'elles ont de leur propre maladie et ainsi contribuer à l'acceptation de celle-ci.

\section{DÉPISTAGE ET TRAITEMENT ADÉQUAT DE LA DÉPRESSION}

La dépression non spécifiée actuelle est significativement plus présente chez les patients du groupe suicide que chez ceux du groupe témoin. Cela appuie la littérature sur le sujet qui démontre une association entre la dépression et le suicide chez les patients atteints de schizophrénie (Caldwell et Gottesman, 1992; Kelly et al., 2005; Kuo et al., 2005 ; Potkin et al., 2003; Rossau et Mortensen, 1997 ; Tejedor, et al., 1987). Cependant, la dépression est souvent non détectée par les médecins qui interprètent souvent ces symptômes dépressifs comme étant des symptômes négatifs de la schizophrénie (Saarinen et al., 1990), ce qui fait en sorte que la dépression, n'étant pas dépistée, n'est pas traitée chez ces patients. Les médecins et professionnels de la santé mentale devraient être plus sensibilisés à la présence de symptômes dépressifs chez leurs patients schizophrènes, surtout chez ceux ayant de la difficulté à accepter leur maladie. Souvent, ces symptômes de dépression sont présents au début du développement de la maladie (Häfner, 2005; Häfner et al., 2005a, Häfner et al., $2005 b)$ et durant une rechute de psychose (an der Heiden et al., 2005). Si la dépression ou les symptômes dépressifs étaient détectés et traités adéquatement durant ces périodes d'extrême vulnérabilité au suicide, cela pourrait diminuer le risque suicidaire chez ces patients.

PRÉPARATION ADÉQUATE DE LA SORTIE DE L'HÔPITAL ET SUIVI ÉTROIT DES

PATIENTS SUIVANT L'HOSPITALISATION

Les résultats sur les événements de vie associés à la maladie soulèvent l'importance du contact avec des professionnels de la santé précédant le suicide: les patients décédés par suicide avaient eu un nombre significativement plus élevé d'hospitalisations ou de séjours en centre de crise au cours des douze derniers mois que les patients du groupe témoin. Cela suggère que les patients suicidés avaient un nombre important de contacts avec des professionnels de la santé durant la dernière année de leur vie. La littérature indique qu'un grand nombre de suicides ont lieu dans les semaines ou les mois suivant la sortie de l'hôpital (Caldwell et Gottesman, 1992; Pompili et al., 2005; Rossau et Mortensen, 1997). Pompili et ses collègues (2005) proposent des stratégies de prévention de suicide où le personnel soignant de l'hôpital peut jouer un rôle actif dans le dépistage des patients schizophrènes avant qu'ils ne sortent de l'hôpital. Ce dépistage inclut l'évaluation de l'environnement dans lequel se retrouvera le patient, une fois sorti de l'hôpital (résidence familiale, appartement supervisé, etc.). Ces stratégies de prévention sont d'autant plus importantes que les patients schizophrènes sont 34 fois plus à risque de se suicider suivant la sortie de l'hôpital que la population en général (Pompili et al., 2005).

INCLUSION DES FAMILLES DANS LE TRAITEMENT DE LEUR PROCHE MALADE

Les professionnels de la santé mentale intervenant auprès de patients atteints de schizophrénie travaillent souvent avec leur famille. Nos résultats ont révélé que ni l'acceptation ni l'implication familiale n'affectaient le risque suicidaire chez les patients. Comment alors les familles peuvent-elles aider leur proche malade? Puisque nos résultats démontrent que les facteurs externes, dont le support/rejet parental, ont moins d'impact sur le suicide que les facteurs internes, propres au patient, sur le suicide, les parents pourraient être impliqués dans le traitement de leur proche d'une autre façon, Les professionnels de la santé mentale pourraient inclure les parents dans le processus thérapeutique afin d'aider leurs enfants adultes atteints de schizophrénie à accepter leur maladie et leur traitement et, par conséquent, à développer un rapport plus positif face à 
leur maladie. Plusieurs études ont démontré que la thérapie impliquant la famille de la personne malade (ex.: des groupes de soutien aux familles) aide à améliorer l'adhérence au traitement chez le patient (Carrà et al., 2007). La psychoéducation familiale aide à réduire le taux d'hospitalisations chez le patient ainsi que le nombre de jours passés à l'hôpital (Bäuml et al., 2006) pouvant améliorer ainsi le fonctionnement social et personnel (désir d'obtenir un emploi, maintien de leurs intérêts, gestion de leurs conflits) du patient (Magliano et al., 2006).

\section{Bibliographie}

AMADOR, X.F., J.M. HARKAVY-FRIEDMAN, C. KASAPIS, S.A. YALE, M. FLAUM et J.M. GORMAN (1996). «Suicidal behavior in schizophrenia and its relationship to awareness to illness ", American Journal of Psychiatry, vol. 153, p. 1185-1188.

AMADOR, X.F., D.H. STRAUSS, S.A. YALE, M.M. FLAUM, N.C. ANDREASEN, S.A. YALE, S.C. CLARK et J.M. GORMAN (1994). "Awareness of illness in schizophrenia and schizoaffective and mood disorders ", Archives of General Psychiatry, vol. 51, $\mathrm{n}^{\circ} 10$, p. 826-836.

AN DER HEIDEN, W., R. KÖNNECKE, K. MAURER, D. ROPETER et H. HÄFNER (2005). "Depression in the long-term course of schizophrenia ", European Archives of Psychiatry and Clinical Neuroscience, vol. 255, p. $174-184$

BIRCHWOOD, M. et P. CHADWICK (1997). "The omnipotence of voices: Testing the validity of a cognitive model », Psychological medicine, vol. 27, $\mathrm{n}^{\circ} 6$, décembre, p. 13451353

BÄUML, J., T. FROBÖSE, S. KRAEMER, M. RENTROP et G. PITSCHEL-WALTZ (2006). «Psychoeducation: A basic psychotherapeutic intervention for patients with schizophrenia and their families", Schizophrenia Bulletin, vol. 32, p. S1-S9.

BIRCHWOOD, M., R. MASON, F. MACMILLAN et J. HEALY (1993). «Depression, demoralization and control over psychotic illness: A comparison of depressed and nondepressed patients with a chronic psychosis ", Psychological Medicine, vol. 23, p. 387-395.

BOLTON, C., P. GOODING, N. KAPUR, C. BARROWCLOUGH et N. TARRIER (2007). «Developing psychological perspectives of suicidal behaviour and risk in people with a diagnosis of schizophrenia: We know they kill themselves but do we understand why? ", Clinical Psychology Review, vol. 27, p. 511-536.

BOURGEOIS, M., J. SWENDSEN, F. YOUNG, X. AMADOR, S. PINI, G.B. CASSANO, J.-P. LINDENMAYER, C. HSU, L. ALPHS, H.Y. MELTZER et THE INTERSEPT STUDY GROUP (2004). "Awareness of disorder and suicide risk in the treatment of schizophrenia : results of the international suicide prevention trial ", American Journal of Psychiatry, vol. 161, p. 1494-1496
BREED, W. (1967). "Suicide and loss in social interaction ", dans E. SHNEIDMAN (dir.), Essays in Self-Destruction, New York, Science House Inc., p. 188-202.

BROWN, G.W., T.O. HARRIS et A. BIFULCO (1989). Life Events and Difficulties Schedule. Londres, Royal Holloway College.

BURKER, E., D.M. EVON, M.M. LOSIELLE, J.B. FINKEL et M.R. MILL (2005). " Coping predicts depression and disability in heart transplant candidates ", Journal of Psychosomatic Research, vol. 59, p. 215-222.

CALDWELL, C.B. et I.I. GOTTESMAN (1992). "Schizophrenia - A high-risk factor for suicide : Clues to risk reduction », Suicide and Life-Threatening Behavior, vol. 22, n० 4 , p. 479-493.

CARRÀ, G., C. MONTOMOLI, C. MASSIMO et C.L. CAZZULLO (2007). «Family interventions for schizophrenia in Italy : Randomized controlled trial», European Archives of Psychiatry and Clinical Neuroscience, vol. $257, \mathrm{n}^{\circ} 1$, p. $23-30$.

CHAN-HYUNG, K., K. JAYATHILAKE et H. MELTZER (2003). « Hopelessness, neurocognitive function, and insight in schizophrenia: Relationship to suicidal behaviour», Schizophrenia Research, vol. 60, p. 71-80.

DE HERT, M., K. MCKENZIE et J. PEUSKE (2001). "Risk factors for suicide in young people suffering from schizophrenia: A long-term follow-up study », Schizophrenia Research, vol. 47, p. 127-134.

FENTON, W.S. (2000). "Depression, suicide and suicide prevention", Suicide and Life-Threatening Behavior, vol. 30, $\mathrm{n}^{\circ} 1$, p. 34-39.

FENNIG, S., N. HORESH, D. ALONI, A. APTER, A. WEIZMAN et S. FENNIG (2005). "Life events and suicidality in adolescents with schizophrenia ", European Childhood and Adolescent Psychiatry, vol. 14, p. $454-460$

FIALKO, L., D. FREEMAN, P.E. BEBBINGTON, E. KUIPERS, P.A. GARETY, G. DUNN et D. FOWLER (2006). «Understanding suicidal ideation in psychosis: Findings from the Psychological Prevention of Relapse in Psychosis (PRP) trial ", Acta Psychiatrica Scandinavica, vol. 114, p. 177-186.

FIRST, M.B., M. GIBBON, J.B.W. WILLIAMS, et B. LORNA (1995). Structured Clinical Interview for DSM-IV Axis II Personality Disorders (SCID-II), New York, Biometrics Research Department, New York State Psychiatric Institute.

FIRST, M.B., M. GIBBON, R.L. SPITZER et J.B.W. WILLIAMS (2001). Structured Clinical Interview for DSM-IV-TR Axis I Disorders Research Version, Patient Edition with Psychotic Screen, New York, Biometrics Research Department, New York State Psychiatric Institute.

FLEISCHHACKER, W.W., H. HÄFNER, J. LEFF, J.J. LOPEZ-LBOR, M. MAJ, N. SARTORIUS, C.N. STEFANIS, R. WARNER et N.N. WIG (1998). "The WPA global programme against stigma and discrimination because of schizophrenia", Schizophrenia Research, vol. 29, $\mathrm{n}^{\circ} 1$, p. 194-194.
GIRÒN, M. et M. GÒMEZ-BENEYTO (1998). "Relationship between empathic family attitude and relapse in schizophrenia : A 2-year follow-up prospective study ", Schizophrenia Bulletin, vol. 24, no 4, p. 619-627.

GREENHOUSE, W., B. MEYER et S.L. JOHNSON (2000). « Coping and medication adherence in bipolar disorder », Journal of Affective Disorders, vol. 59, p. 237-241.

HAAS, G.L. (1997). "Suicidal behavior in schizophrenia », dans R. MARIS, M. SILVERMAN, et S. CANETTO (dir.), Review of Suicidology, p. 203-236.

HARKAVY-FRIEDMAN, J.M., K. RESTIFO, D. MALASPINA, C.A. KAUFMANN, X.F. AMADOR, S.A. YALE et J.M. GORMAN (1999). "Suicidal behavior in schizophrenia: Characteristics of individuals who had and had not attempted suicide», American Journal of Psychiatry, vol. 156, p. 1276-1278.

HAWTON, K., L. APPLEBY, S. PLATT, T. FOSTER, J. COOPER, A. MALMBERG et S. SIMKIN (1998). "The psychological autopsy approach to studying suicide: A review of methodological issues ", Journal of Affective Disorders, vol. 50, p. 269-276.

HAWTON, K., L. SUTTON, C. HAW, J. SINCLAIR et J.J. DEEKS (2005). «Schizophrenia and suicide: Systematic review of risk factors », British Journal of Psychiatry, vol. 187, p. 9-20.

HÄFNER, H. (2005). "Schizophrenia and depression ", European Archives of Clinical Neuroscience, vol. 255, p. 157-158.

HÄFNER, H., K. MAURER, G. TRENDLER, W. AN DER HEIDEN et M. SCHMIDT (2005a). «The early course of schizophrenia and depression », European Archives of Clinical Neuroscience, vol. 255, p. 167-173.

HÄFNER, H., K. MAURER, G. TRENDLER, W. AN DER HEIDEN, M. SCHMIDT et R. KÖNNECKE (2005b). «Schizophrenia and depression: Challenging the paradigm of two separate diseases - A controlled study of schizophrenia, depression and healthy controls », Schizophrenia Research, vol. 77, p. 11-24.

HEÏLA, H., E.T. ISOMETSÄ, M.M. HENRIKSSON, M.E. HEIKKINEN, M.J. MARTTUNEN et J.U.K. LÖNNQVIST (1997). "Suicide and schizophrenia: A nationwide psychological autopsy study on age- and sexspecific clinical characteristics of 92 suicide victims with schizophrenia », American Journal of Psychiatry, vol. 154, no 9, p. 1235-1242.

HEÏLA, H., E.T. ISOMETSA, M.M. HENRIKSSON, M.E. HEIKKINEN, M.J. MARTTUNEN et J.K. LÖNNQVIST (1998). "Antecedents of suicide in people with schizophrenia », British Journal of Psychiatry, vol. 173, p. 330-333.

HEÏLA, H., M.E. HEIKKINEN, E.T. ISOMETSÄ, M.M. HENRIKSSON, M.J. MARTTUNEN et J.K. LÖNNQVIST (1999). «Life events and completed suicide in schizophrenia: A comparison of suicide victims with and without schizophrenia ", Schizophrenia Bulletin, vol. 25, no 3, p. 519-531.

HUNT, I., N. KAPUR, K. WINDFUHR, J. ROBINSON, H. BICKLEY, S. FLYNN, R. PARSONS, J. BURNS, J. SHAW et 
L. APPLEBY. (2006). « Suicide in schizophrenia: Findings from a national clinical survey », Journal of Psychiatric Practice, vol. 12, no 3, p. 139-147.

INSKIP, H.M., E.C. HARRIS et B. BARRACLOUGH (1998). "Lifetime risk of suicide for affective disorder, alcoholism and schizophrenia », British Journal of Psychiatry, vol. 172 , p. 35-37.

ISOMETSA, E.T. (2001). «Psychological autopsy studies - A review », European Psychiatry, vol. 16, p. 379-385.

KANEDA, Y. (2005). "Suicidality in schizophrenia as a separate symptom domain that may be independent of depression or psychosis : Letter to the editors ", Schizophrenia Research, vol. 81, p. 113-114.

KELLY, D.L., J. SHIM, S.M. FELDMAN, Y. YU et R.R. CONLEY (2005). "Lifetime psychiatric symptoms in persons with schizophrenia who died by suicide compared to other means of death ", Journal of Psychiatric Research, vol. 38, p. 531536.

KIM, C.-H., K. JAYATHILAKE et H. MELTZER (2003). «Hopelessness, neurocognitive function, and insight in schizophrenia: Relationship to suicidal behavior », Schizophrenia Research, vol. 50, p. 71-80.

KING, S. et M. DIXON (1999). «Expressed emotion and relapse in young schizophrenia outpatients », Schizophrenia Bulletin, vol. 25, $\mathrm{n}^{\mathrm{0}} 2$, p. 337-386.

KUO, C.-J., S-Y. TSAI, C.-H. LO, Y-P. WANG et C-C. CHEN (2005). «Risk factors for completed suicide in schizophrenia ", Journal of Clinical Psychiatry, vol. 66, no 5, p. 579-585.

LEUCHT, S. et S. HERES (2006). «Epidemiology, clinical consequences, and psychosocial treatment of nonadherence in schizophrenia », Journal of Clinical Psychiatry, vol. 67, suppl. 5, p. 3-8.

LESAGE, A.D., R. BOYER, F. GRUNBERG, C. VANIER, R. MORISSETTE, C. MENARDBUTEAU et M. LOYER (1994). "Suicide and mental disorders: A case-control study of young men", The American Journal of Psychiatry, vol. 151, p. 1063-1068.

LESTER, D. (2006). «Sex differences in completed suicide by schizophrenic patients: A meta-analysis », Suicide and Life-Threatening Behavior, vol. 36, ${ }^{\circ} 1$, p. 50-56.

MAGLIANO, L., A. FIORILLO, C. MALANGONE, C. DE ROSA, M. MAJ et THE FAMILY INTERVENTION GROUP (2006). "Patient functioning and family burden in a controlled, real-world trial of family psychoeducation for schizophrenia », Psychiatric Services, vol. 57, $\mathrm{n}^{\circ}$ 12, p. 1784-1791.

MCGIRR, A., M. TOUSIGNANT, D. ROUTHIER, L. POULIOT, N. CHAWKY, H. MARGOLESE et G. TURECKI (2006). « Risk factors for completed suicide in schizophrenia and other chronic psychotic disorders: A case-control study», Schizophrenia Research, vol. 84, p. 132-143.

MISHARA, B. et M. TOUSIGNANT (2004). Comprendre le suicide, Québec, Presses de l'Université de Montréal.

MIKLOWITZ, D.J. (2004). «The role of family systems in severe and recurrent psychiatric disorder: A developmental psychopathology view», Development and Psychopathology, vol. 16, p. 667-688.

PALMER, B.A., V.S. PANKRATZ et J.M. BOSTWICK (2005). «The lifetime risk of suicide in schizophrenia : A reexamination ", Archives of General Psychiatry, vol. 62, n 3, p. 247-253.

PERÄLÄ, J., J. SUVISARI, S.I. SAARNI, K. KUOPPASALMI, E. ISOMETSÄ, S. PIRKOLA, T. PARTONEN, A. TUULIOHENRIKSSON, J. HINTIKKA, T. KIESEPPÄ, T. HÄRKÄNEN, S. KORSKINEN, et J. LÖNNQVIST (2007). " Lifetime prevalence of psychotic and bipolar I disorders in a general population », Archives of General Psychiatry, vol. 64, p. 19-28.

PHELAN, J.C., B.G. LINK, A. STUEVE et B.A. PESCOLIDO (2000). «Public conceptions of mental illness in 1950 and 1996 : What is mental illness and is it to be feared? ", Journal of Health and Social Behavior, vol. 41, $\mathrm{n}^{\circ} 2$, p. 188-207.

POLLACK, L. (1995). «Striving for stability with bipolar disorders ", Archives of Psychiatric Nursing, vol. 9, n 3, p. 122-129.

POMPILI, M, X.F. AMADOR, P. GIRARDI, J. HARKAVY-FREEDMAN, M. HARROW, K. KAPLAN, M. KRAUSZ, D. LESTER, H.Y. MELTZER, J. MODESTIN, L.P. MONTROSS, P.B. MORTENSEN, P. MUNKJORGENSEN, J. NIELSEN, M. NORDENTOFT, P.I. SAARINEN, S. ZISOOK, S.T. WILSON, et R. TATARELLI (2007). "Suicide risk in schizophrenia: Learning from the past to change the future », Annals of General Psychiatry, vol. 6, p. 10.

POMPILI, M., I. MANCINELLI, A. RUBERTO, G.D. KOTZALDIS, P. GIRARDI et R. TATARELLI (2005). "Where schizophrenic patients commit suicide: A review of suicide among inpatients and former inpatients ", International Journal of Psychiatry in Medicine, vol. 35, $\mathrm{n}^{\circ}$ 2, p. 171-190.

POMPILI, M., A. RUBERTO, G.D. KOTZALIDID, P. GIRARDI et R. TATARELLI (2004). "Suicide and awareness of illness in schizophrenia: An overview », Bulletin of the Menninger Clinic, vol. 68, $\mathrm{n}^{\circ}$ 4, p. 297-318.

POTKIN, S.G., L. ALPHS, C. HSU, K.R.R. KRISHNAN, R. ANAND, F.K. YOUNG, F.K., H. MELTZER, A. GREEN et THE INTERSEPT STUDY GROUP (2003). «Predicting suicidal risk in schizophrenic and schizoaffective patients in a prospective two-year trial », Biological Psychiatry, vol. 54, p. 444-452.

POULIOT, L. et D. DE LEV (2006). "Critical Issues in Psychological Autopsy Studies», Suicide \& Life-Threatening Behavior, vol. 36, $\mathrm{n}^{\circ}$ 5, p. 491-510.

PUIG-ANTICH, J., H. ORVASHEL, M.A. TABRIZI et W.J. CHAMBER (1995). Kiddie-SADS-E (K-SADS-E), Montréal, Hôpital Rivière-des-Prairies, Service de Recherche.

RECASENS, C., L. MIMIC, R. DARDENNES, J.D. GUELFI et F. ROUILLON (2002). «Conscience de soi et conscience de la maladie dans la schizophrénie: relations avec l'anxiété et la dépression », Annales Médico Psychologiques, vol. 160, p. 589-595.

ROOKE, O. et M. BIRCHWOOD (1998). «Loss, humiliation and entrapment as appraisals of schizophrenic illness: A prospective study of depressed and non-depressed patients », British Journal of Clinical Psychology, vol. 37, p. 259-268.

ROSSAU, C.D. et P.B. MORTENSEN (1997). "Risk factors for suicide in patients with schizophrenia : Nested case-control study », British Journal of Psychiatry, vol. 171, p. 355359.

ROY, A. (1982). «Suicide in chronic schizophrenia », British Journal of Psychiatry, vol. 141, p. 171-177.

ROY, A., A. MAZONSON et D. PICKAR (1984). «Attempted suicide in chronic schizophrenia » British Journal of Psychiatry, vol. 144, p. 303-306.

RUSSELL, A.J. et J.L. BROWNE (2005). «Staying well with bipolar disorder», Australian and New Zealand Journal of Psychiatry, vol. 39, p. 187-193.

SAARINEN, P.I., J. LEHTONEN et J. LÖNNQVIST (1999). "Suicide risk in schizophrenia: An analysis of 17 consecutive suicides », Schizophrenia Bulletin, vol. 25, n ${ }^{\circ} 3$, p. 533-542.

SARTORIUS, N. (2007). «Stigma and mental health. Commentary», The Lancet, vol. 370, $\mathrm{n}^{\circ} 9590$, p. $810-811$.

SCHULZE, B. et M.C. ANGERMEYER (2003). "Subjective experiences of stigma. A focus group study of schizophrenic patients, their lives and mental health professionals ", Social Science and Medicine, vol. 56, p. 299312

SHNEIDMAN, E. (1993). Suicide as Psychache: A Clinical Approach to Self-Destructive Behavior, New Jersey, Jason Aronson Inc.

STUART, H. (2003). « Stigmatisation. Leçons tirées des programmes visant sa diminution ", Santé mentale au Québec, vol. 28, $\mathrm{n}^{\circ} 1$, p. 54-72.

TEJEDOR, M.C., J.J. CASTILLON, J.M. PERICAY, M. PUIGDELLIVOL et E. TURNES (1987). "Suicidal behavior in schizophrenia», Crisis : Journal of Crisis Intervention and Suicide, vol. 8, $\mathrm{n}^{\circ}$ 2, p. 151-161.

WARD, A., K. ISHAK, I. PROSKOROVSKY et J. CARO (2006). "Compliance with refilling prescriptions for atypical antipsychotic agents and its association with the risks for hospitalization, suicide, and death in patients with schizophrenia in Quebec and Saskatchewan: A retrospective database study ", Clinical Therapeutics, vol. 28, n 11, p. 19121921.

WILLIAMS, M. (1997). Cry of pain, Understanding Suicide and Self-Harm, Ontario, Penguin Books.

YEN, C., M. YEH, C. CHEN et H. CHUNG (2002). "Predictive value of insight for suicide, violence, hospitalization, and social adjustment for outpatients with schizophrenia: A prospective study», Comprehensive Psychiatry, vol. 43, n 6, p. 443-447. 